Rückblick

\title{
Wie kommt das Huhn ins CT? Viel zu entdecken bei den MTRA-Aktionstagen
}

„Schau rein, was für Dich drinsteckt“: Im so genannten X-Ray-Monat November luden bundesweit 15 MTRA-Schulen und radiologische Institute Jugendliche ein, die Ausbildung zum MedizinischTechnischen Radiologieassistenten (MTRA) zu erleben. Insgesamt folgten rund $\mathbf{5 0 0}$ Interessierte der Einladung und wurden mit Röntgenbilderrätseln, Live-Vorführungen an den radiologischen Großgeräten und informativen Vorträgen begrüßt.

Besonders begeistert waren die jungen Besucherinnen und Besucher über den direkten Austausch mit den engagierten MTRASchülerinnen und -Schülern, Lehrern und Radiologen vor Ort. Sie zeigten die Tätigkeitsfelder eines/einer MTRA, beispielsweise in der radiologischen Diagnostik, aber auch in der Dosimetrie und Strahlenkunde sowie in der Nuklearmedizin und Strahlentherapie. Das Programm, das die meisten

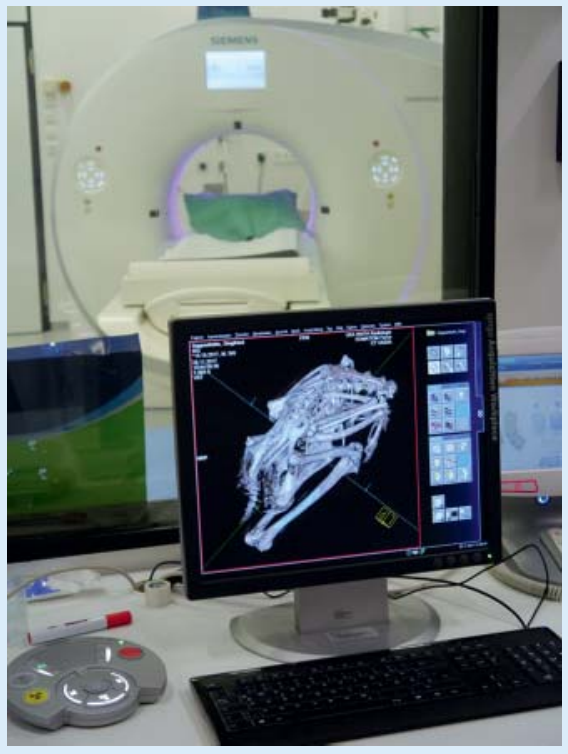

Am Beispiel von „Suppenhuhn Siegfried“ lernten die jungen Besucherinnen und Besucher, wie ein CT funktioniert. Foto: Uniklinik RWTH Aachen/Ingrid Offenhäusser

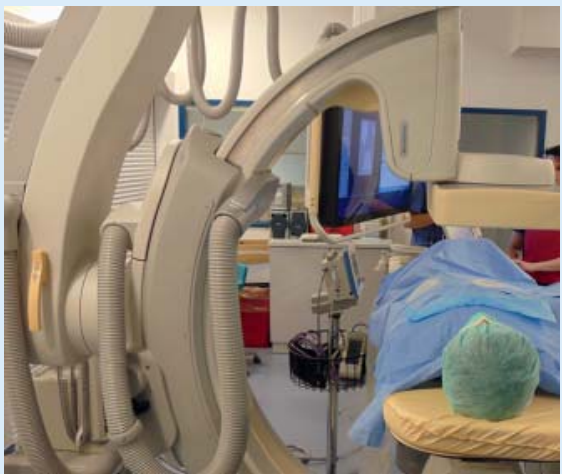

Hier kommt das lebensgroße Phantom zum Einsatz. Foto: MTRA-Schule Universitätsklinikum Bonn

MTRA-Schulen an einem Samstag anboten, gab damit praxisorientierte Einblicke in den Arbeitsalltag. Anschaulich wurden Abläufe an realen Arbeitsplätzen erklärt und in Vorträgen erläutert, wie die Ausbildung an der jeweiligen Schule abläuft und welche Weiterbildungsmöglichkeiten MTRA haben. 
Wie anspruchsvoll und vielfältig der Beruf des/der MTRA ist, zeigt sich bereits in der sehr praxisorientierten Ausbildung. So müssen die angehenden Experten nicht nur lernen, wie man mit den hochtechnologischen Geräten wie beispielsweise einem Magnetresonanztomografen (MRT) oder einem Röntgengerät umgeht, sondern auch viel menschliches Einfühlungsvermögen mitbringen, da sie in engem Kontakt mit den Patienten stehen.

Ob Kinosaal mit MTRA-Info-Film oder ein Überraschungsei im Computertomografen - überall an den teilnehmenden Schulen

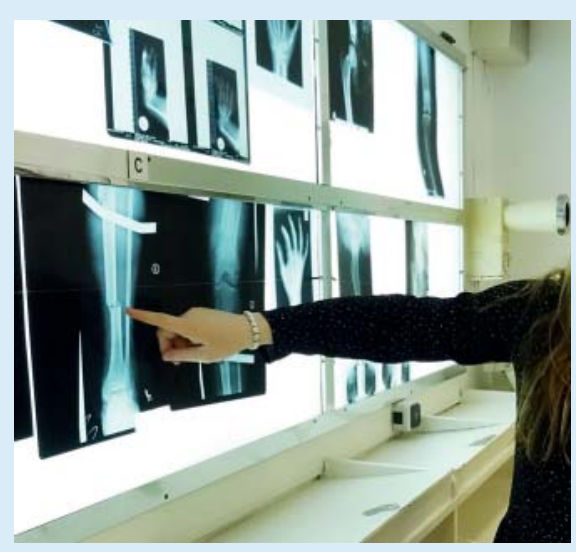

Entdeckt! Die Fraktur im Röntgenbilderrätsel. Foto: MTA-Schule für Radiologie, Asklepios Bildungszentrum für Gesundheitsberufe Hamburg

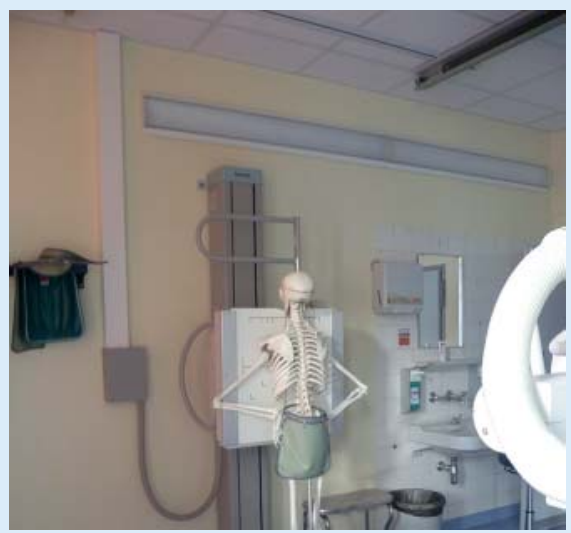

So funktioniert Strahlenschutz. Foto: Berufsfachschule für medizinisch-technische Radiologieassistenten - Kh/MTRA, Klinikum Nürnberg und den kooperierenden Kliniken gab es etwas zu entdecken. „Besonders das CT mit dem Hühnchen hat mir gefallen“, schreibt eine Besucherin in ihrem Feedback zum Aktionstag der MTA-Schule Aachen. Die Gäste durften aber auch selbst aktiv werden: das Aufziehen von Spritzen ausprobieren, eine Maske für die Strahlentherapie anfertigen oder ein Röntgenbild von einem lebensgroßen Phantom anfertigen. Der Einsatz der MTRA-Schulen zeigte Wirkung: Viele Besucherinnen und Besucher gaben noch während des Aktionstags ihre Bewerbung zur/zum MTRA ab. 